\title{
Bypass besser als Stent?
}

Erst Anfang Oktober hatte der aktuelle Herzbericht es dokumentiert: Verengte Herzkranzgefäße werden immer öfter durch Einsetzen eines Stents behandelt. Die Stent-Implantation an Herzkranzgefäßen wurde im Jahr 2010 in Deutschland 325872 Mal durchgeführt, eine Zunahme um 5\% gegenüber 2009. Die „Koronare Bypass-Operation“ hingegen wurde in 2010 nur in 55993 Fällen durchgeführt. Dass dieser Trend zur vermeintlich schonenderen Stentimplantation längerfristig gesehen für die Patienten mit erheblichen Nachteilen einhergeht, zeigen die 4-Jahres-Ergebnisse der weltweit größten vergleichenden Studie zwischen den beiden Behandlungsformen. Die Studie wurde auf dem jährlichen Kongress der europäischen Herzchirurgen in Lissabon vom Studienleiter, dem niederländischen Kardiologen Professor Patrick Serruys, erstmals vorgestellt. Danach sollten Patienten mit mittleren und schweren Herzkranzgefäßerkrankungen die Bypass-Operation der Stentimplantation vorziehen, da sie die klar bessere Alternative mit Blick auf das Überleben des Eingriffs und das erkrankungs- freie Leben nach dem Eingriff ist. Die Ergebnisse der internationalen Studie „Synergie between PCI with taxus and Cardiac Surgery“ (Syntax) auf Basis von rund 1800 Patienten dokumentieren, dass die koronare Bypass-Operation den medikamentenbeschichteten Stents hinsichtlich der Sterblichkeit, der Häufigkeit eines nachfolgenden Herzinfarkts und der Notwendigkeit eines wiederholten Eingriffs deutlich überlegen ist. Während 4 Jahre nach dem Eingriff mehr als 91\% der Bypass-Patienten noch leben, sind zu diesem Zeitpunkt fast $12 \%$ der mit einem Stent behandelten Patienten verstorben. Noch deutlicher sind die Unterschiede bei der Lebensqualität. So sind nach Stentimplantationen mehr als doppelt so oft erneute Eingriffe als nach einer Bypass-Operation notwendig $(23,9 \%$ vs. $11,9 \%)$ und die Rate der Herzinfarkte nach einer Stentimplantation ist mit 8,3\% deutlich höher als die nach einer Bypass-Operation mit 3,8 \%. "Auf die Zahlen des Herzberichtes übertragen heißt dies, dass in Deutschland zum Nachteil der Patienten zu häufig die Entscheidung für einen Stent statt für die Bypass-
Operation getroffen wird“, kommentierte Professor Friedrich Wilhelm Mohr, Präsident der Deutschen Gesellschaft für Thorax-, Herz- und Gefäßchirurgie (DGTHG), die aktuellen Studienergebnisse. „Bei differenzierter Bewertung der vorliegenden Studienerkenntnisse finden sich für $\mathrm{Pa}-$ tienten mit wenig komplexer Verengung der Herzkranzgefäße vergleichbare Ergebnisse für die die koronare Bypass-Operation und die Stentimplantation, so dass beide Verfahren hierfür geeignet sind. Dagegen ist für Patienten mit komplexen Erkrankungsformen wie der Verengung aller Herzkranzgefäße die Todesfallrate 4 Jahre nach Stentimplantation dreimal so hoch wie die nach einer koronaren Bypassoperation, so dass bei diesen Patienten die Bypassoperation deutlich vorteilhafter ist. Die aktuellen Ergebnisse der Syntax-Studie bekräftigen damit eindrucksvoll die Festlegungen der 'Nationalen Versorgungsleitlinie chronische KHK' zur Behandlung von Patienten mit koronarer Herzerkrankung, die gemeinsam mit den Fachgesellschaften von Herzchirurgen und Kardiologen bereits im Jahr 2006 herausgegeben wurde. Zum Wohle der Patienten sind Kardiologen und Herzchirurgen aufgefordert, diese Leitlinie deutlich konsequenter als bisher in der Praxis anzuwenden."

Idw/EACTS 2011, Lissabon 